\title{
Can Incomplete Metastasectomy Impact Renal Cell Carcinoma Outcomes? A Propensity Score Matching Analysis From a Prospective Multicenter Collaboration
}

\author{
Alice Dragomir, ${ }^{* 1}$ Charles Hesswani, ${ }^{* 1}$ Gautier Marcq, ${ }^{1}$ Alan I. So, ${ }^{2}$ Christian Kollmannsberger, ${ }^{2}$ \\ Naveen S. Basappa, ${ }^{3}$ Adrian Fairey, ${ }^{3}$ Anil Kapoor, ${ }^{4}$ Aly-Khan A. Lalani, ${ }^{4}$ Antonio Finelli, ${ }^{5}$ Lori A. Wood, ${ }^{6}$ \\ Daniel Y.C. Heng, ${ }^{7}$ Georg Bjarnason, ${ }^{8}$ Rodney H. Breau, ${ }^{9}$ Luke T. Lavallée, ${ }^{9}$ Denis Soulières, ${ }^{10}$ \\ Darrel Drachenberg, ${ }^{11}$ Frédéric Pouliot, ${ }^{12}$ Simon Tanguay $\bowtie 1$ \\ ${ }^{*}$ Co-first authors \\ ${ }^{1}$ McGill University, Montreal, Canada ${ }^{2}$ Department of Urologic Sciences, University of British Columbia, Vancouver, Canada ${ }^{3}$ Cross Cancer Institute, University of \\ Alberta, Edmonton, Canada ${ }^{4}$ Juravinski Cancer Centre, McMaster University, Hamilton, Canada 5 Princess Margaret Cancer Centre, University of Toronto, Toronto, \\ Canada ${ }^{6}$ Queen Elizabeth II Health Sciences Centre, Halifax, Canada ${ }^{7}$ Tom Baker Cancer Centre, University of Calgary, Calgary, Canada ${ }^{8}$ Odette Cancer Centre, \\ Sunnybrook Health Sciences Centre, Toronto, Canada ${ }^{9}$ Ottawa Hospital Cancer Center, Ottawa, Canada ${ }^{10}$ Centre Hospitalier de I'Université de Montréal, Montreal, \\ Canada ${ }^{11}$ University of Manitoba, Winnipeg, Canada ${ }^{12}$ Centre Hospitalier Universitaire de Québec, Université Laval, Quebec, Canada
}

\section{Abstract}

Objectives To evaluate the role of incomplete metastasectomy (IM) for patients with metastatic renal cell carcinoma (mRCC) on overall survival (OS) and time to introduction of first-line systemic therapy.

Methods Patients diagnosed with mRCC between January 2011 and April 2019 in 16 centers were selected from the Canadian Kidney Cancer information system database. We included mRCC patients who had prior nephrectomy and had received an IM (resection of at least 1 metastasis) or no metastasectomy (NM). A propensity score matching was performed to minimize selection bias. Cox proportional hazards analysis was used to assess the impact of the metastasectomy while adjusting for potential confounders. OS was assessed by Kaplan-Meier analysis.

Results A total of 138 patients with mRCC underwent IM, while 1221 patients did not. On multivariate analysis, IM did not improve OS (hazard ratio [HR] $0.96,95 \%$ CI 0.63 to $1.45, P=0.836$ ) However, subgroup analyses revealed IM improved OS compared with NM when lungs were the only site involved (median time to OS not reached versus 66 months, respectively; $P=0.014$ ). Additionally, lung metastasectomy delayed the systemic therapy compared with $\mathrm{NM}$ (median 41 and 13 months, respectively, $P=0.014$ ). IM of endocrine organs (thyroid, pancreas, adrenals) or bone metastases did not impact OS.

Conclusion The role of IM for mRCC is limited. Incomplete resection of lung metastases was associated with improved OS and delayed time to introduction of systemic therapy when lungs were the sole location of metastatic disease. Despite case-matching, unknown unadjusted confounders may explain the relationship between IM and survival in this analysis.

\section{Key Words}

Kidney neoplasms, incomplete metastasectomy, neoplasm metastasis, clear-cell metastatic renal cell carcinoma, systemic therapy

\section{Competing Interests}

None declared

\section{Article Information}

Received on September 27, 2020

Accepted on January 2, 2021

Soc Int Urol J. 2021;2(2):82-95

DOI: https://doi.10.48083/WQFR32352

Correction: This article was amended on March 19, 2021, to correct errors in author names. 


\section{Abbreviations}

CM complete metastasectomy

HR hazard ratio

IM incomplete metastasectomy

IOR interquartile range

mRCC metastatic renal cell carcinoma

$\mathrm{NM}$ no metastasectomy

OS overall survival

RCC renal cell carcinoma

\section{Introduction}

In 2020, kidney cancer was estimated to be the sixth most common neoplasm in Canadian men and the eighth most common in Canadian women[1]. Renal cell carcinoma (RCC) is metastatic at diagnosis in 15\% of cases, and metastasis will occur after diagnosis in up to $30 \%$ of patients[2]. Despite the advent of systemic therapies and immunotherapies, drug resistance remains a major therapeutic challenge in treating metastatic RCC. Since current systemic therapy does not offer complete response in most patients with metastatic disease, surgical resection of metastases can be used to improve survival. Most studies have compared complete metastasectomy (CM) with incomplete or no metastasectomy, and demonstrated a significant improvement in overall survival (OS) $[3,4]$. A recent systematic review pooling 8 studies and 2267 patients demonstrated an improved OS in patients undergoing CM when compared with those who had incomplete metastasectomy (IM) or no metastasectomy (NM) (OS 36.5 to 142 months for CM and 8.5 to 27 months for IM/NM)[5]. Similarly, a recent single institution retrospective cohort study comparing patients undergoing CM with those who had IM/NM demonstrated a significantly improved OS (at 2 years $81 \%$ versus $53 \%, P<0.001)[6]$.

Few studies have compared the outcomes of IM patients with those of NM patients. The few available studies have been discordant and/or published before the era of tyrosine kinase inhibitors and immunotherapy[7-9]. All were single-center studies with limited power to allow extrapolation and clear recommendations for daily practice. Multicenter studies comparing IM with NM are lacking. The present study was conducted to assess OS and time to first-line systemic therapy of IM in patients with metastatic RCC (mRCC) when compared to patients who did not receive metastasectomy in a large prospective multicenter database using propensity score matching.

\section{Methods}

Patient demographics and clinical and pathological data were collected from the Canadian Kidney Cancer information system (CKCis) database, a prospective multicenter collaboration of 16 institutions in 6 Canadian provinces. Baseline information was obtained from patient surveys and the medical record and included age, sex, date of RCC and mRCC diagnosis, date of nephrectomy, comorbidities, and the location and number of metastases. CM was defined as surgical resection of all visible metastases, while IM was defined as resection of some, but not all, of the radiographically visible metastases. IM was performed without curative intent. If this information was missing, the no evidence of disease status of the patient post-metastasectomy was used to classify patients between the IM and the $\mathrm{CM}$ groups. Complete versus incomplete resection was specified by patient's medical records. If the patient underwent subsequent metastasectomy at different dates, the CM versus IM status was defined on the first metastasectomy. The indication for the metastasectomy and the reason for incomplete surgical resection were not available. Research Ethics Board approval was obtained at each individual participating center.

Medical records of patients diagnosed with mRCC who underwent radical or partial nephrectomy between January 2011 and April 2019 were reviewed. Patients receiving a $\mathrm{CM}$ were excluded and were reported separately[10]. Histopathological evaluation of the nephrectomy specimen was used to diagnose RCC. The date of diagnosis of a first metastasis confirmed by imaging was labeled as the index date. The analysis ranged from the index date to the end of follow-up, defined as the earliest date between the last patient visit, the date of death, or the end of study period (April 31, 2019).

Clinical characteristics including sex, age, number and location of metastases, metastasectomy sites, pathological stage, clear cell histology, synchronicity, comorbidities (diabetes mellitus, hypertension, dyslipidemia, cardiovascular disease, smoking status, and obesity), Charlson comorbidity index score (excluding the solid tumors) and time from RCC diagnosis until metastasis were identified at the index date. "Endocrine" metastasis was defined as a metastasis at one of the following sites: adrenal glands, pancreas, and thyroid. The use of systemic therapy and radiation therapy was assessed at the index date and during follow-up. A propensity score method was used to determinate the predicted probability of receiving an IM based on clinical characteristics evaluated at index date including age $(<65$ years versus $\geq 65)$, sites and number of metastasis ( 1 versus $\geq 2$ ), synchronous disease 
(yes versus no) and clear cell histology (yes versus no). For each patient, a propensity score and its quintiles were obtained. In order to reduce selection bias, patients receiving IM were matched with patients who had not received a metastasectomy. Up to 4 patients were randomly selected for each patient with IM. The date of IM or the date of selection was defined as the matching date. The matching was performed for (1) the propensity score quintiles (ie, probability of receiving an IM), (2) the status of the systemic treatment at the time of matching (yes versus no), (3) time between the first metastasis and the RCC diagnosis ( $<3$ months, 3 months to $<6$ months, 6 months to $<1$ year, 1 year to $<2$ years, and $\geq 2$ years), and (4) an equivalent duration of follow-up between index date and the matching date.

Clinical and demographic characteristics between IM group and NM group were performed using $t$ test for continuous variables and chi-square test for categorical variables. Individual propensity scores of IM were obtained using a multivariate logistic regression model based on variables enumerated in the section above. Overall survival was calculated from matching date to death from any cause. Kaplan-Meier curve analysis was performed to estimate OS since matching date in the matched cohort, with log-rank test to compare the IM and NM groups. Similarly, the time to introduction of systemic therapy during follow-up was assessed in IM and NM groups. The Cox proportional hazards regression model was used in the matched cohort to evaluate the association between IM and overall survival by adjusting for different covariables that were not used for matching. These included sex, age 65 and older (yes versus no), sites and number of sites of metastasis and clear cell carcinoma versus other histology. The use of systemic treatment and the use of radiation therapy were also included as time-dependent factors. A second Cox model was performed by adding an additional factor, the Charlson comorbidity index ( 0 or 1 versus $>1$ ).

To assess differences between IM and NM by metastatic sites we performed several stratified analyses. Kaplan-Meier curve analyses were performed (1) to estimate OS since matching date and (2) to estimate time to introduction of systemic therapy during follow-up, in the matched cohort, with log-rank test to compare the IM and NM groups by metastatic site (lung, lymph nodes, bones, and endocrine). Patients with brain and liver metastasis were included in the multiple sites analysis but were not considered from the individual sites analyses because of the low number of patients in these groups. Finally, Kaplan-Meier curve was performed to assess the OS among patients receiving IM by metastasectomy sites. All statistical tests were 2 -sided, with a $P<0.05$ considered significant. All analyses were performed using SASS (version 9; SAS Institute, Cary, North Carolina).

\section{Results}

During the study period, the CKCis cohort included 8936 patients diagnosed with RCC (Figure 1); 6223 patients did not develop metastasis and were therefore excluded from the analysis.

Patients without prior nephrectomy (336 patients) or who had received a CM (266 patients) were also excluded from the cohort. Our final cohort consisted of 1367 patients: 146 of these patients underwent an IM, and 1221 patients did not undergo any form of metastasectomy (Figure 1).

The demographics and clinicopathological characteristics of the cohort before propensity score matching were summarized in Supplementary Table S1. One hundred thirty-eight patients who underwent an IM were matched with 522 patients with NM based on usage of systemic therapy before selection or date of IM, delay between primary tumor diagnosis and first metastasis, and propensity score quintiles (Table 1).

\section{FIGURE 1.}

Flowchart

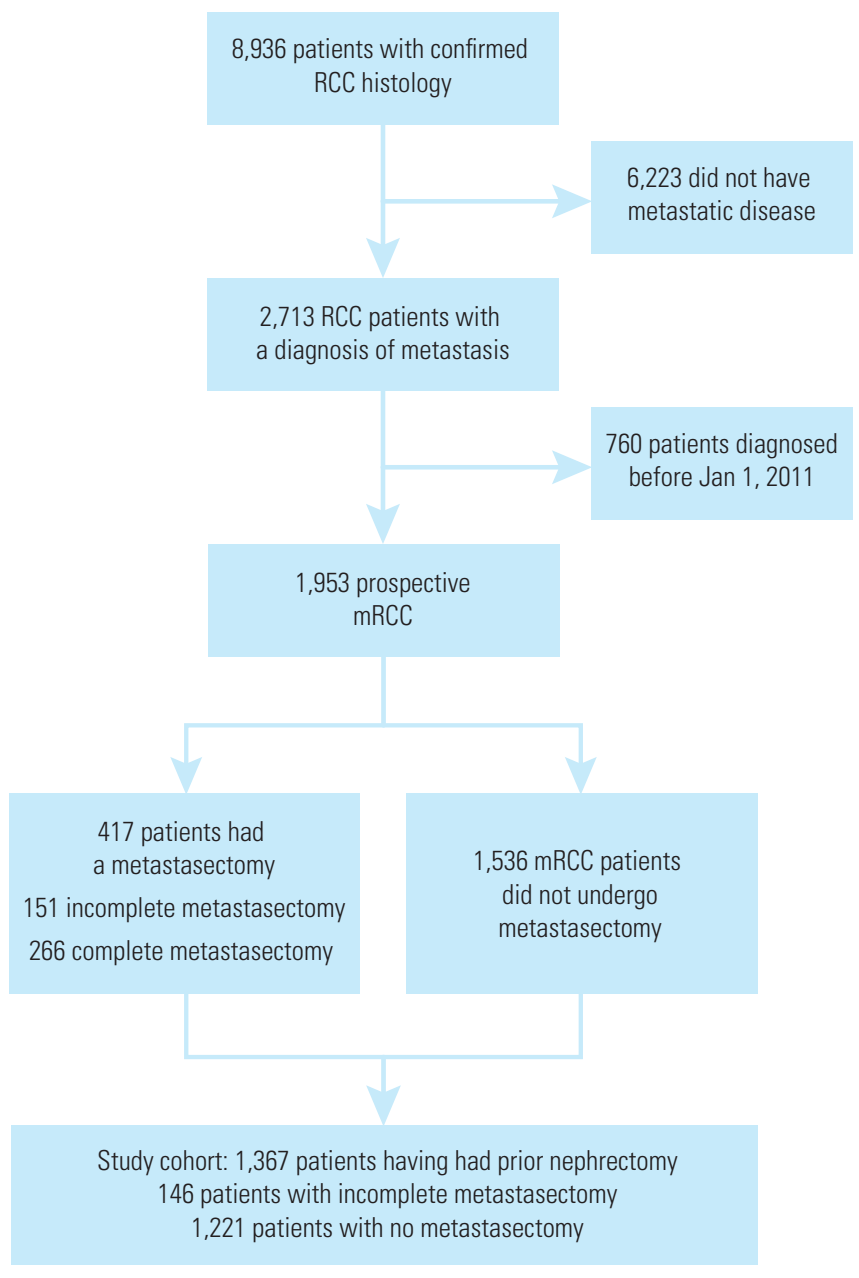


TABLE 1.

Patient demographics, matched cohort

\begin{tabular}{|c|c|c|c|}
\hline & $\begin{array}{l}\text { Incomplete } \\
\text { metastasectomy }\end{array}$ & $\begin{array}{l}\text { No metastasectomy } \\
\text { (matched with incomplete) }\end{array}$ & P Value \\
\hline No. patients & 138 & 522 & \\
\hline Median age at diagnosis (IQR) & $63.5(56-69)$ & $65(57-72)$ & $0.1496^{a}$ \\
\hline Over 65 years old at mRCC diagnosis, $\%$ & 43.5 & 51.2 & 0.109 \\
\hline Male, \% & 79.0 & 73.6 & 0.193 \\
\hline Female, \% & 21.0 & 26.4 & \\
\hline Median follow-up, months (IOR) & $26(16-54)$ & $26(12-42)$ & $0.058^{a}$ \\
\hline $\begin{array}{l}\text { Time between primary tumor to metastasis, median } \\
\text { (IQR) (months) }\end{array}$ & $14.9(0-60)$ & $14.3(1-48)$ & $0.376^{a}$ \\
\hline Over 1 year from primary tumor to metastasis, \% & 59.2 & 52.9 & 0.981 \\
\hline \multicolumn{4}{|l|}{$\begin{array}{l}\text { Time between the index date }{ }^{\mathrm{b}} \text { and the time of } \\
\text { matching }{ }^{\mathrm{c}} \text { date, median (IOR) (months) }\end{array}$} \\
\hline \multicolumn{4}{|l|}{ Propensity score quintiles } \\
\hline First & 72.5 & 74.1 & \multirow{3}{*}{0.558} \\
\hline Second & 24.6 & 24.3 & \\
\hline Third & 2.9 & 1.5 & \\
\hline
\end{tabular}

\section{Pathological T-stage at diagnosis, \%}

\begin{tabular}{|c|c|c|c|c|}
\hline & $\mathrm{T} 1$ & 25.4 & 31.7 & \multirow{5}{*}{0.002} \\
\hline & T2 & 16.7 & 15.5 & \\
\hline & T3 & 45.7 & 48.9 & \\
\hline & T4 & 5.8 & 2.5 & \\
\hline & Tx & 6.5 & 1.4 & \\
\hline \multicolumn{2}{|l|}{ Clear cell RCC (yes vs no), \% } & 84.8 & 82.8 & 0.579 \\
\hline \multicolumn{2}{|l|}{ Synchronous metastasis (yes vs no), \% } & 39.9 & 40.2 & 0.936 \\
\hline \multicolumn{2}{|l|}{ Metachronous metastasis (yes vs no), \% } & 60.1 & 59.8 & \\
\hline \multicolumn{2}{|l|}{ Had a nephrectomy (yes vs no), \% } & 100 & 100 & - \\
\hline \multicolumn{5}{|l|}{ Number of organ sites with metastasis } \\
\hline \multicolumn{2}{|r|}{1} & 71.0 & 82.4 & 0.003 \\
\hline \multicolumn{2}{|c|}{$\geq 2$} & 29.0 & 17.6 & \\
\hline $\begin{array}{l}\text { First-line systemic treatment } \\
\text { prior to matching datec }(\%)\end{array}$ & & 16.7 & 15.7 & 0.784 \\
\hline
\end{tabular}

${ }^{a}$ Wilcoxon two-sample test for medians. ${ }^{b}$ Index date: the date of the first metastasis. ${ }^{c}$ Matching date: the date of incomplete metastasectomy or the date of selection. ${ }^{d}$ Patients with multiple locations were counted in all specific locations. ${ }^{e}$ Fisher exact text. 


\section{TABLE 1.}

Patient demographics, matched cohort, Cont'd

\begin{tabular}{|c|c|c|c|}
\hline & $\begin{array}{l}\text { Incomplete } \\
\text { metastasectomy }\end{array}$ & $\begin{array}{l}\text { No metastasectomy } \\
\text { (matched with incomplete) }\end{array}$ & $P$ Value \\
\hline \multicolumn{4}{|l|}{ Sites of metastasis, $\mathrm{d} \%$} \\
\hline Lung & 41.3 & 44.6 & 0.483 \\
\hline Bones & 26.8 & 26.6 & 0.965 \\
\hline Liver & 8.7 & 6.1 & 0.283 \\
\hline Brain & 5.1 & 2.3 & 0.083 \\
\hline Endocrine & 15.9 & & 0.04 \\
\hline \multicolumn{4}{|l|}{ Systemic treatment during follow-up, \% } \\
\hline First-line (ref: yes) & 71.1 & 63.4 & 0.068 \\
\hline Second-line (ref: yes) & 33.0 & 31.7 & 0.763 \\
\hline $\begin{array}{r}\text { Radiation treatment at index date and } \\
\text { during follow-up (ref: yes), \% }\end{array}$ & 56.0 & 42.0 & 0.004 \\
\hline \multicolumn{4}{|l|}{ Comorbidities, \% } \\
\hline Hypertension (ref: yes) & 55.1 & 35.6 & $<0.0001$ \\
\hline Diabetes (ref: yes) & 29.0 & 13.4 & $<0.0001$ \\
\hline Hypercholesterolemia (ref: yes) & 23.9 & 13.6 & 0.003 \\
\hline Coronary artery disease & 8.0 & 9.0 & 0.703 \\
\hline Cardiovascular disease & 11.6 & 8.1 & 0.190 \\
\hline Smoker (ref: yes) & 4.4 & 0 & $<0.0001^{e}$ \\
\hline Obesity (ref: yes) & 3.6 & 3.3 & $0.831^{e}$ \\
\hline \multicolumn{4}{|l|}{ Charlson Score index, \% $(n=624)$} \\
\hline 0 or 1 & 66.7 & 49.3 & \multirow{2}{*}{0.0004} \\
\hline More than 1 & 33.3 & 50.7 & \\
\hline
\end{tabular}

${ }^{a}$ Wilcoxon two-sample test for medians. ${ }^{b}$ Index date: the date of the first metastasis. ${ }^{c}$ Matching date: the date of incomplete metastasectomy or the date of selection. ${ }^{d}$ Patients with multiple locations were counted in all specific locations. ${ }^{e}$ Fisher exact text.

All the variables used in the matching were balanced after matching the IM group and their respective NM patients. After matching, several other features remained or were balanced between the 2 groups, such as age over 65 years, sex, more than 1 year from primary tumor diagnosis and usage of systemic therapy during follow-up (Table 1). A significant difference persisted among the pathological stages at diagnosis between each group. The IM group had a higher percentage of $\mathrm{pT} 2$, pT4 and pTx, while the NM group had increased pT1 and pT3 disease $(P=0.002)$. More patients received radiation treatment in the IM group than in the NM group (56.0 versus $42 \%, P=0.036)$. The number of organs involved by metastasis was also significantly different. In the IM group, $71 \%$ of patients had a single organ with metastases compared with $82.4 \%$ of patients in the NM group $(P=0.003)$. More patients in the IM group had hypertension $(P<0.001)$, diabetes mellitus $(P<$ $0.001)$, dyslipidemia $(P=0.031)$, and history of smoking $(P<0.001)$. One-third $(33.3 \%)$ of patients in the IM had a Charlson comorbidity index score over 1 compared with $50.7 \%$ of patients in the NM group $(P=0.004)$ (Table 1$)$.

The median OS was similar between the IM group and their matched NM group (58 months IQR 22 to "not reached" [NR]) versus 47 months IQR (18 to NR); $P=0.878$ ), respectively (Figure 2A). At 12 months, $81.2 \%$ 


\section{FIGURE 2A.}

Overall survival for patients with IM vs NM all organ sites combined (matched cohort)

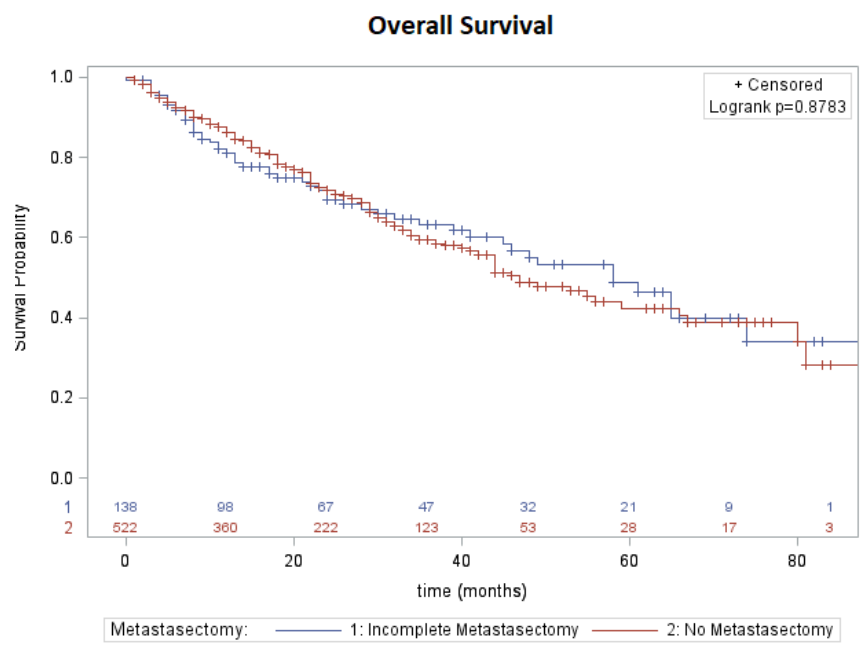

FIGURE 2B.

Overall survival for patients with IM vs NM all organ sites combined (matched cohort)

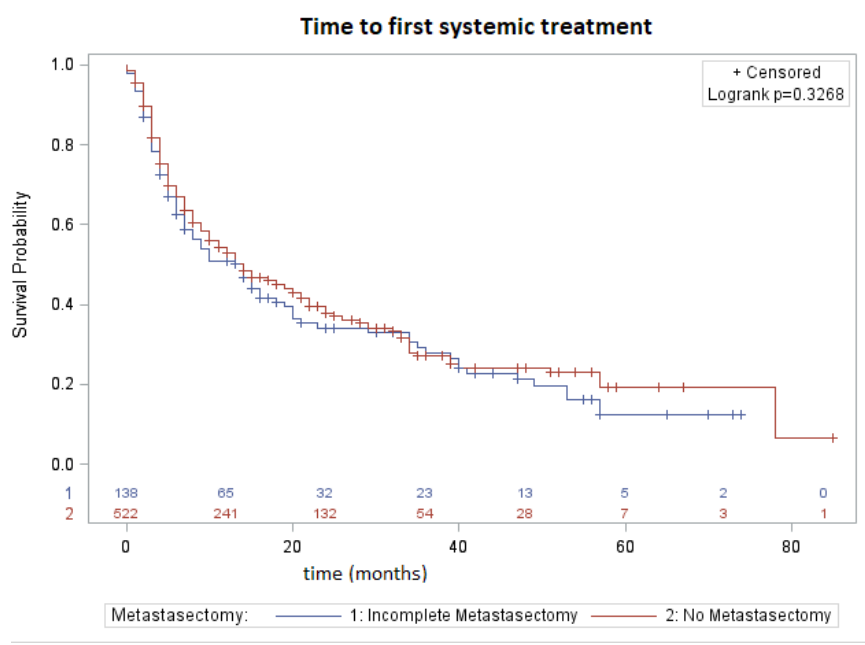

of patients were still alive in the IM group, and $86.2 \%$ in its matched NM group. The 5-year OS was $48.7 \%$ versus $42.5 \%$, respectively (Figure $2 \mathrm{~A}$ ). Regarding the time to introduction of systemic therapy, no significant difference was observed between the IM and the NM group $(P=0.327)$ (Figure 2B).

The multivariate analysis revealed that performing an IM was not associated with a survival advantage compared with NM (hazard ratio [HR] 0.96, 95\% CI 0.63 to $1.45, P=0.836$ ) (Table 2). Clear cell histology was not found to be an independent factor associated with a decreased risk of mortality compared with other histology (HR $0.63,95 \%$ CI 0.38 to $1.041, P=0.071$ ). When including the Charlson comorbidity index in the multivariate analysis, the risk of mortality related to an IM remained similar (HR 1.05, 95\% CI 0.0 .69 to 0.1 .60 ) (data not shown). Having a Charlson comorbidity score of 0 or 1 versus more than 1 , was not associated with a reduced risk of death (HR $0.89,95 \%$ CI 0.56 to 1.41 ).

Stratified analyses by site of metastasis revealed that IM in patients with only lung metastases, led to improved OS (median OS not reached versus 66 months in the NM group) (2-year OS 82\% versus 68\%, 5 -year OS $82 \%$ versus $50 \%$, respectively) $(P=0.026)$ (Figure 3A). IM of bone or endocrine lesions did not show a significant difference in survival compared with NM $(P=0.849$ and $P=0.388$, respectively), while IM of lymph nodes showed a decreased OS compared with NM (median OS 14 months versus NR) (2-year OS $44.4 \%$ versus $73.8 \%$ ) (Figure $3 \mathrm{~A}$ ). IM of liver and brain lesions was not assessed because of the low number of events at these sites. Furthermore, analysis performed for the time to systemic treatment initiation revealed that only IM in patients with lung metastasis resulted in a delayed initiation of systemic therapy (median of 41 months (95\% CI 14 to 57) compared with 13 months (95\% CI 7 to 35) in NM patients) $(P=0.026)$ (Figure 3B). No significant difference was found for bone, lymph node or endocrine metastasectomy $(P=0.063, P=0.149$ and $P=0.701$, respectively) (Figure $3 \mathrm{~B}$ ).

While there was no difference in OS observed between the IM and NM groups all organ sites combined, the OS differed among IM sites (Supplementary Figure 1A). Among patients with an IM, those with lung lesions had better survival (2-year OS of $86 \%$; 5 -year OS of $86 \%$ ) (median not reached) when compared with patients who had bone, lymph node, and endocrine metastasis (2-year OS $62 \%, 44 \%$ and $76.8 \%$; 5 -year OS $35 \%$, NA and $57.6 \%$, respectively) (medians 41 months (IQR 11 to 65), 14 months (IQR 8 to NR), and 61 months (IQR 46 to NR), respectively) $(P=0.004)$ (Supplementary Figure 1A).

Subgroup analysis of IM patients with a single organ site involved by metastasis yielded a significant difference between groups $(P=0.003)$ The IM of lung lesions had the higher delay to first-line systemic therapy initiation when compared to bone, lymph node, and endocrine metastasectomy (Supplementary Figure 1B). The median time to systemic therapy initiation was 39 months (IQR 14 to 57) compared with 5 months (IQR 3 to 19 ), 8 months (IQR 3 to NA), and 15 months (IQR 6 to 57$)$, respectively.

\section{Discussion}

The role of IM on overall survival in mRCC patients, as part of a multimodal treatment approach, is limited. IM continues to be performed, however, mainly for palliative reasons. Also, IM and stereotactic body radiation therapy are being increasingly used for 
TABLE 2.

Cox regression model incomplete metastasectomy versus no metastasectomy

\begin{tabular}{|c|c|c|c|c|}
\hline Variables & $\begin{array}{l}\text { Univariate } \\
\text { HR (95\%CI) }\end{array}$ & $P$ Value & $\begin{array}{l}\text { Multivariate } \\
\text { HR (95\%CI) }\end{array}$ & $\boldsymbol{P}$ Value \\
\hline N (metastasectomy vs. no metastasectomy) & 138 vs. 522 & - & 138 vs. 522 & - \\
\hline Had a metastasectomy & $1.03(0.71-1.51)$ & 0.868 & $0.96(0.63-1.45)$ & 0.836 \\
\hline Male (ref: female) & $0.90(0.62-1.31)$ & 0.580 & $0.92(0.62-1.38)$ & 0.686 \\
\hline $\begin{array}{l}\text { Systemic therapy during follow-up } \\
\text { (time-dependent variable) (ref: yes) }\end{array}$ & $0.87(0.57-1.35)$ & 0.545 & $0.91(0.56-1.46)$ & 0.685 \\
\hline Age (ref: $\geq 65$ years) & $1.42(1.01-2.00)$ & 0.044 & $1.39(0.96-2.02)$ & 0.079 \\
\hline Clear cell renal cell carcinoma (ref: yes) & $0.62(0.39-0.97)$ & 0.038 & $0.63(0.38-1.04)$ & 0.071 \\
\hline Bones metastasis (ref: yes) & $1.76(0.88-3.54)$ & 0.110 & $1.58(0.67-3.77)$ & 0.299 \\
\hline Liver metastasis (ref: yes) & $1.18(0.60-2.32)$ & 0.634 & $1.59(0.70-3.62)$ & 0.2699 \\
\hline Lung metastasis (ref: yes) & $1.12(0.73-1.71)$ & 0.616 & $1.52(0.84-2.73)$ & 0.165 \\
\hline Brain metastasis (ref: yes) & $0.98(0.22-4.36)$ & 0.977 & $0.84(0.15-4.68)$ & 0.8399 \\
\hline Lymph node metastasis (ref: yes) & $1.41(0.75-2.65)$ & 0.281 & $1.72(0.86-3.41)$ & 0.0 .124 \\
\hline Endocrine metastasis (ref: yes) & $1.24(0.61-2.50)$ & 0.554 & $1.40(0.66-2.98)$ & 0.381 \\
\hline More than 1 location of tumor (ref: 1 location) & $1.22(0.80-1.84)$ & 0.359 & $0.79(0.39-1.60)$ & 0.513 \\
\hline Radiation therapy (time-dependent variable) (ref: yes) & $1.51(1.01-2.25)$ & 0.05 & $1.49(0.96-2.3)$ & 0.076 \\
\hline
\end{tabular}

Ref: reference.

oligoprogression. In our cohort, $10.7 \%$ of patients underwent an IM, a rate comparable to those found in other studies (range $9 \%$ to $19 \%$ ) [7,11-13]. The median age of patients at the time of metastasis is also similar to that in other studies (61 to 65 years old) $[14,15]$. In addition, our analysis consisted of primarily clear cell histology $(84.8 \%)$, which is similar to the findings of previous studies $(80 \%$ to $93.8 \%)[7,16,17]$. After matching, most clinicopathologic characteristics were similar between the 2 groups, with the exception of pathological T-stage, number of organ sites involved by metastasis, and comorbidities. More patients had 2 and more organ sites involved by metastasis in the IM than the NM. Additionally, patients having undergone IM had more comorbidities than NM patients; however, Charlson score favored the IM group. This finding may reflect the existence of poorly controlled or more severe comorbidities in the NM group, resulting in healthier patients being selected for surgery. However, when adjusting for the Charlson comorbidity index, the HR of IM versus NM remained the same, and this was not found to be an independent factor associated to the survival in this population.

Our survival analysis showed that there was no significant difference in OS among patients who had undergone IM compared with those who had not $(P=0.8)$. Our results are comparable to those of other studies in which IM had no OS advantage over NM. As stated before, You et al. reported comparable OS medians of 29.6 versus 23.5 months ([95\% CI 15.4 to 43.8 months], and [95\% CI 18.9 to 28.1 months], respectively) for IM and NM, respectively[12]. In addition, Yu and colleagues also reported in a singlecenter retrospective study of patients undergoing IM, 


\section{FIGURE 3A.}

Overall survival for patients with IM vs NM all organ sites combined (matched cohort)
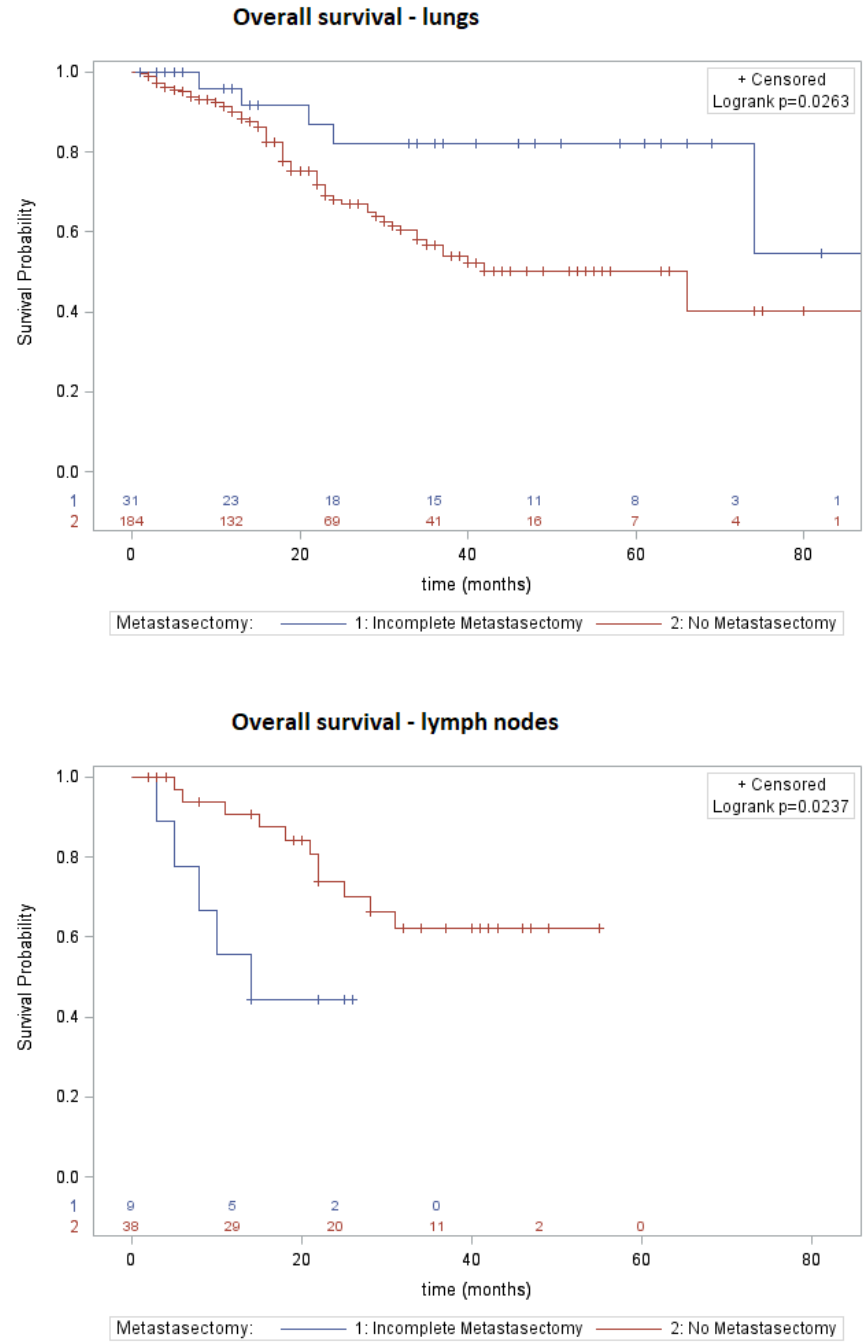

similar OS among IM and NM with median OS of 16 months versus 22 months (95\% CI 9.5 to 22.5 and 17.6 to 26.4 , respectively)[7]. In contrast, our recent study using the CKCis database found a significant improvement in OS all-sites combined when CM was compared to NM (median OS 82 months [95\% CI 80 to NR] versus 66 months [95\% CI 60 to NR], respectively $[P=0.001])[10]$. Although no difference in survival was shown among all metastasectomy sites combined, the risk of death among different IM sites is not similar. Patients with lung and endocrine IM experienced longer survival rates than those with bone and lymph node IM. However, in our subgroup analyses comparing each site to their matched NM, only lung IM was associated with better OS. In fact, lung IM had a 5-year OS of $82 \%$ compared with $50.4 \%$ without surgical resection. These results are similar to what was previously reported in the literature for CM (range $75 \%$ to $83.3 \%$ )[18,19]. Site-specific survival analyses were not conducted in previous
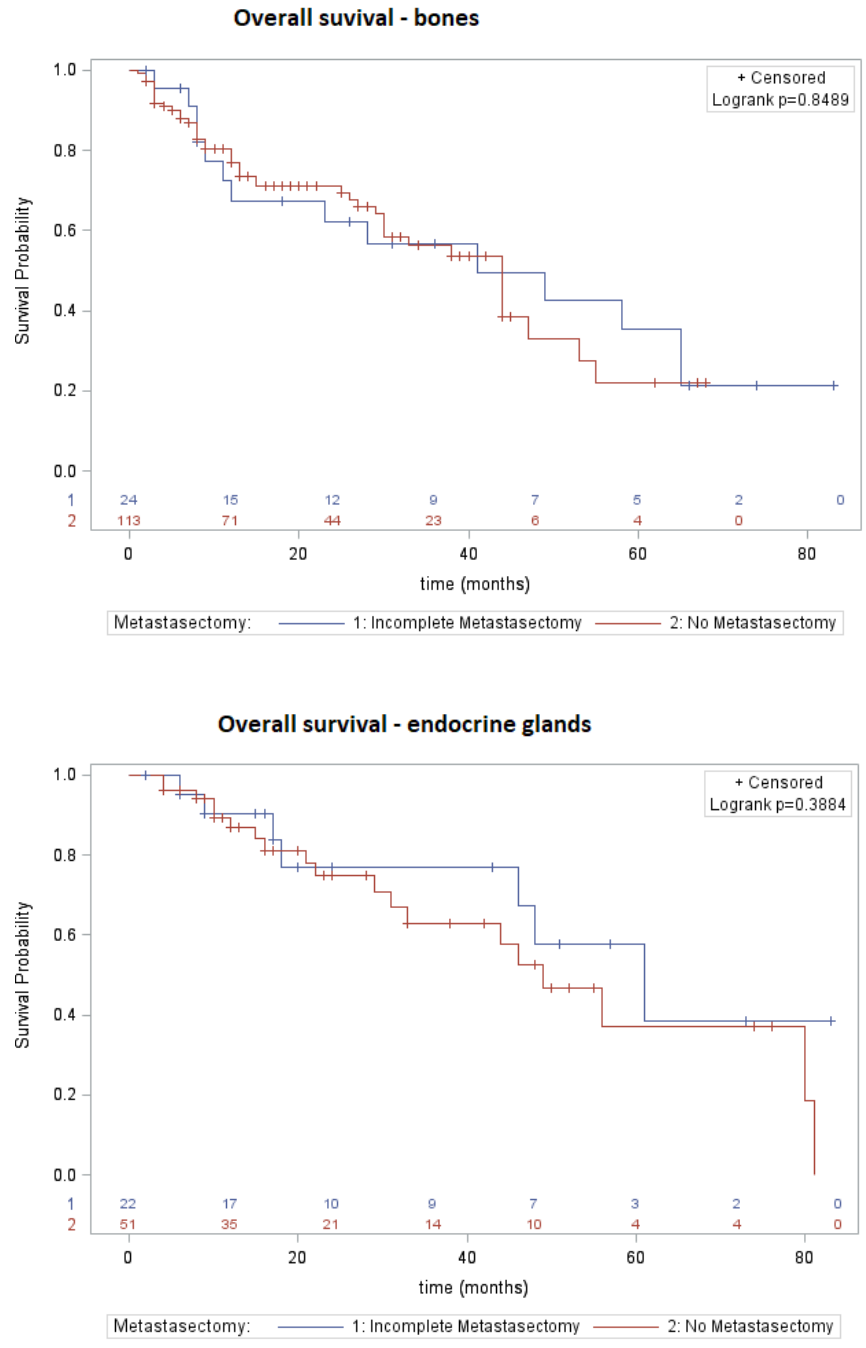

studies evaluating IM[7,8,12]. From what is reported in the literature, lymph nodes, both regional and distant, represent $15 \%$ of sites of metastatic recurrences of RCC[20]. In our study, survival was worsened in patients with incomplete lymphadenectomy when compared with patients without surgical resection $(P=0.024)$. Yet this finding should be interpreted with caution, as this analysis is likely underpowered to assess the role of IM on lymph node metastasis since only 9 patients received an incomplete resection of lymph nodes, and no multivariate analysis was possible. Additionally, lymphadenectomy is often done in conjunction with organ metastasectomy. Our study confirmed that there is no benefit for OS when single or multiple metastatic sites are incompletely resected. Therefore, the role of lymphadenectomy is limited and should not be part of a multi-site IM. Analysis of liver and brain IM was inadequate because of the small number of cases present in our registry ( $8.7 \%$ and $5.1 \%$, respectively). As for 


\section{FIGURE 3B.}

Time to first line systemic treatment for patients with IM vs NM by metastatic location (matched cohort)
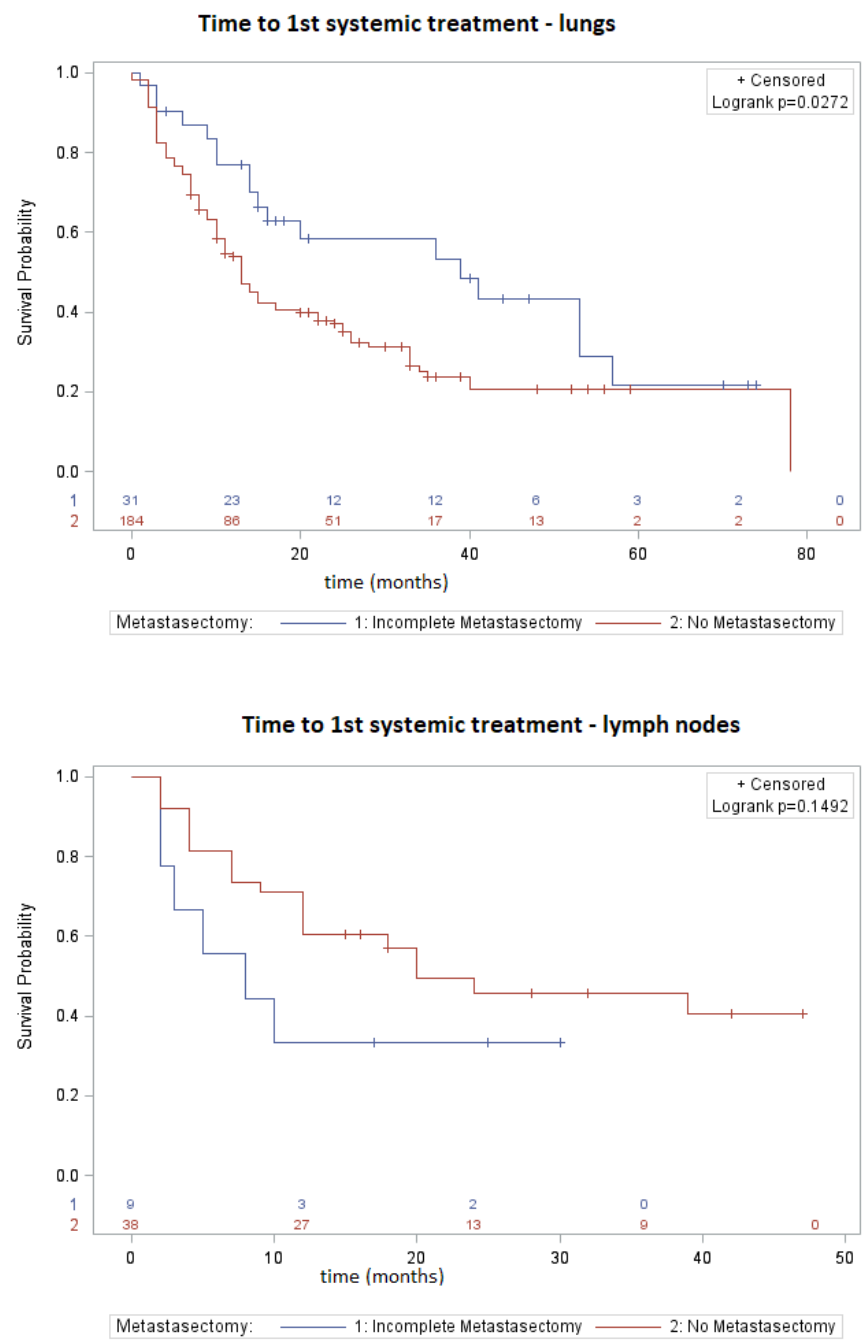

endocrine IM, our results confirmed the absence of survival benefits $(P=0.388)$. Kavolius et al. previously demonstrated that among patients undergoing CM, those with isolated endocrine disease (pancreas, adrenal, ovary, thyroid, and salivary gland) exhibited the most favorable prognosis with a 5 -year OS of 63\%[9]. However, the benefit was only in patients who received a CM and not in those who had an IM or NM.

This study is the first to demonstrate significantly delayed mean time to introduction of first-line systemic therapy (28 months delay in this study) compared with NM. Therefore, when lung lesions represent the only site of metastasis, there may be a benefit of partial resection of metastasis on survival and time to systemic therapy. Given that lungs are the most common organ site for metastasis in renal cell carcinoma, one can speculate that the indication for metastasectomy included a higher proportion of patients with oligoprogression when
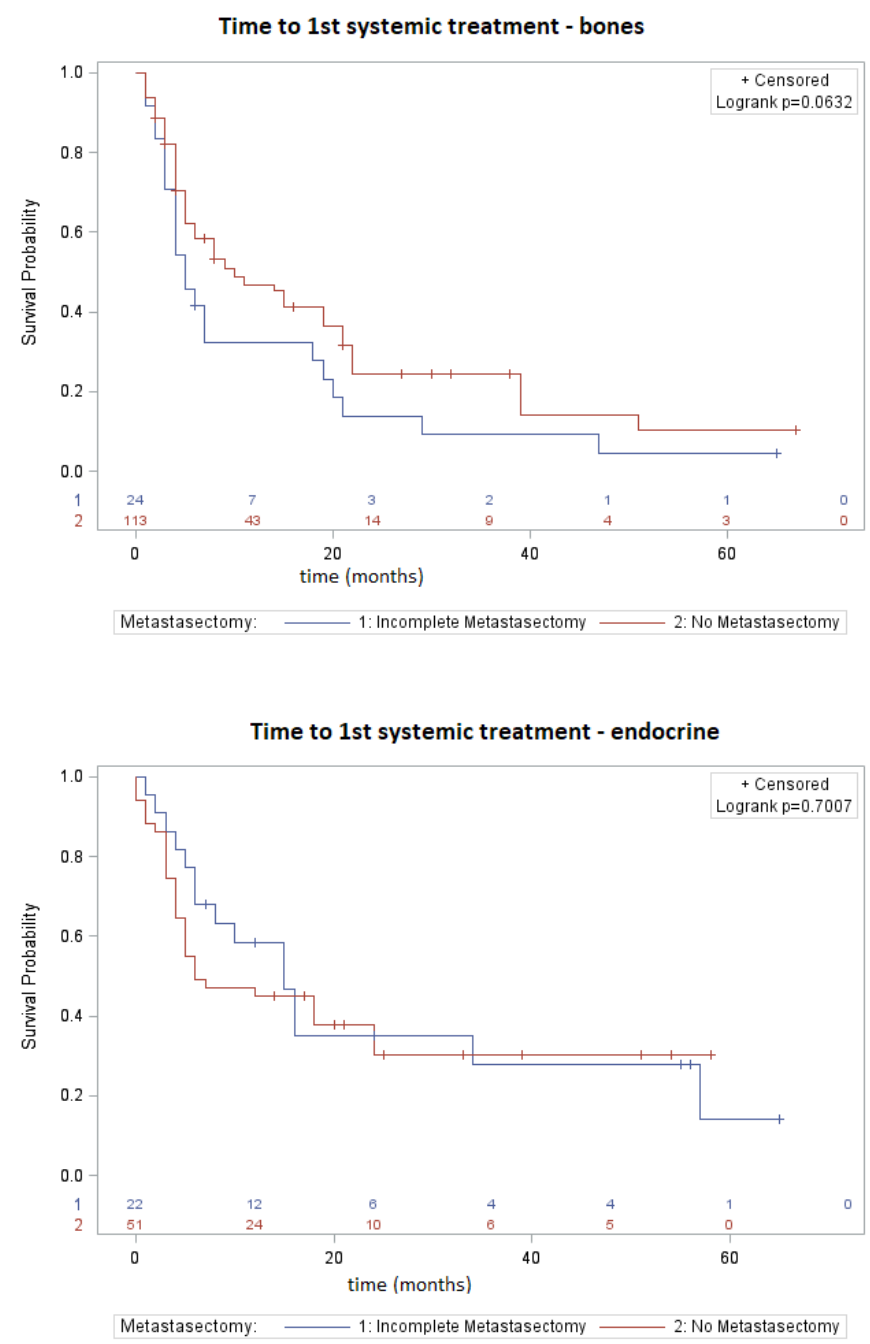

compared with other sites[21]. Metastasectomy at other organ sites, such as bone or brain metastasectomy, may have been performed at a higher proportion for palliative measures such as for pathological fractures or seizures. Unfortunately, it is impossible to confirm this hypothesis through the CKCis database, which constitutes a limitation of our study. While stereotactic body radiation therapy is mainly used in the contemporary era, surgery may represent one treatment modality for such lesions. It is important to note that patients undergoing IM had a higher burden of metastasis and more comorbidities than patients without surgical resection, and despite this, a survival benefit was still evident in subgroup analyses. Incomplete metastasectomy could not be categorized according to the extent of metastasis resected due to the unavailability of this information.

The main limitation of our study arises from its retrospective nature. Also a selection bias may have 
occurred with respect to the patient's characteristics. Indeed, patients with a worse general condition may have been deemed unfit for surgery and were selected in the NM group. We performed a propensity score analysis along with matching analysis to address this limitation. Propensity score estimation was carried for variables such as age, sites and number of metastases, disease synchronicity, and histology, and each patient undergoing incomplete surgical resection was matched with up to 4 patients among those who did not have a metastasectomy for variables deemed associated with treatment choice, including prior use of systemic therapy. This analysis allowed us to balance these clinicopathologic variables and drastically limit selection bias. With the exception of the number organs involved by metastasis, all were balanced in the final cohort. Interestingly, patients in the IM group had a higher percentage of patients with 2 or more organ sites involved than did the NM group. Additionally, patients in the IM group had a higher rate of hypertension, diabetes, and hypercholesterolemia, and were more likely to be smokers. Therefore, despite having more comorbidities and a higher disease burden, a significant improvement in OS and delay to introduction of systemic therapy was obtained for lung IM. Unfortunately, no multivariate analysis was possible because of the limited number of patients in the analysis stratified by metastasis location. Our results show potential benefits of IM for lung metastasis only, compared with NM; however, our multivariate analysis found that having a lung metastasis was not a predictive factor for OS when compared with other metastasis location. Furthermore, a better survival was observed for patients receiving IM for lung metastasis only in comparison with IM for other single metastasis locations such as endocrine, lymph nodes, and bones. Again, the small number of patients in each group made impossible a multivariate analysis or another alternative method, ie, matching strategy. Another limitation is the lack of quality of life $(\mathrm{QoL})$ assessment post-metastasectomy. IM may negatively affect patient's QoL, especially when performed in a nonpalliative setting. Future comparative trials for different modalities for metastasectomy such as stereotactic body radiation therapy versus surgery versus cryotherapy may shed more light on not only potential survival benefits of one modality compared with the other, but also on improvement in patients' QoL. Lastly, the patients' list of medications was unavailable. Some medications, such as metformin or angiotensin converting enzyme inhibitors, may alter survival in metastatic renal cell carcinoma patients[22,23].

One of the main strengths of our analysis is the multicenter prospective registry: our study included 138 patients treated in 16 different hospitals across the country, making this study one of the largest studies on IM.

\section{Conclusion}

Our study indicates that the benefit of IM on OS for mRCC patients is limited. Incomplete resection of lung metastasis shows a potential improvement in OS and delayed time to introduction of systemic therapy when lungs are the sole location of metastatic disease. Outside this indication, IM is not associated with a survival benefit, but may have a role in select patients for symptom relief or palliation. In addition, survival of patients receiving IM varies depending on the metastasectomy site, with the highest value observed for singular lung metastasis. Although groups were matched by propensity score on important measured covariates, some unmeasured confounders may have existed and may have impacted the survival benefit of lung IM in this analysis. Future randomized controlled trials are required to shed more light onto the role of IM. 


\section{References}

1. Siegel RL, Miller KD, Jemal A. Cancer statistics, 2020. CA Cancer J Clin.2020;70(1):7-30.

2. Tajzler C, Tanguay S, Mallick R, Ahrens B, Ly TL, Breau RH, et al. Determining generalizability of the Canadian Kidney Cancer information system (CKCis) to the entire Canadian kidney cancer population. Can Urol Assoc J.2020;14(10). doi: 10.5489/cuaj.6716

3. Breau RH, Blute ML. Surgery for renal cell carcinoma metastases. Curr Opin Urol.2010;20(5):375-81.

4. Dabestani S, Marconi L, Hofmann F, Stewart F, Lam TB, Canfield $\mathrm{SE}$, et al. Local treatments for metastases of renal cell carcinoma: a systematic review. Lancet Oncol.2014;15(12):e549-e61.

5. Zaid HB, Parker WP, Safdar NS, Gershman B, Erwin PJ, Murad MH, et al. Outcomes following complete surgical metastasectomy for patients with metastatic renal cell carcinoma: a systematic review and meta-analysis. J Urol.2017;197(1):44-9.

6. Lyon TD, Thompson RH, Shah PH, Lohse CM, Boorjian SA, Costello $B A$, et al. Complete surgical metastasectomy of renal cell carcinoma in the post-cytokine era. J Urol.2020;203(2):275-82.

7. Yu X, Wang B, Li X, Lin G, Zhang C, Yang Y, et al. The significance of metastasectomy in patients with metastatic renal cell carcinoma in the era of targeted therapy. BioMed Res Int. Published online 2015;0ct. doi: 10.1155/2015/176373

8. Orlova R, Borisov P, Karlov PA, Shkolnik M. Efficacy of incomplete metastasectomy with targeted therapy in patients with metastatic renal cell carcinoma. Am Soc Clin Oncol. 2016;34(15 Suppl). doi: 10.1200/JC0.2016.34.15_suppl.e16117

9. Kavolius JP, Mastorakos DP, Pavlovich C, Russo P, Burt ME, Brady MS. Resection of metastatic renal cell carcinoma. J Clin Oncol.1998;16(6):2261-6.

10. Dragomir A, Nazha S, Wood LA, Rendon RA, Finelli A, Hansen A, et al. Outcomes of complete metastasectomy in metastatic renal cell carcinoma patients: The Canadian Kidney Cancer information system experience. Urol Oncol.2020;38(10):799.e1-799.e10. doi: 10.1016/j.urolonc.2020.07.021.

11. Alt AL, Boorjian SA, Lohse CM, Costello BA, Leibovich BC, Blute ML. Survival after complete surgical resection of multiple metastases from renal cell carcinoma. Cancer.2011;117(13):2873-82.

12. You D, Lee C, Jeong IG, Song C, Lee J-L, Hong B, et al. Impact of metastasectomy on prognosis in patients treated with targeted therapy for metastatic renal cell carcinoma. J Cancer Res Clin Oncol.2016;142(11):2331-8.
13. Vogl U, Zehetgruber H, Dominkus $M$, Hejna M, Zielinski C, Haitel A, et al. Prognostic factors in metastatic renal cell carcinoma: metastasectomy as independent prognostic variable. $\mathrm{Br} J$ Cancer.2006;95(6):691-8.

14. Assouad J, Petkova B, Berna P, Dujon A, Foucault C, Riquet M. Renal cell carcinoma lung metastases surgery: pathologic findings and prognostic factors. Ann Thorac Surg.2007;84(4):1114-20.

15. Hofmann H-S, Neef H, Krohe K, Andreev P, Silber R-E. Prognostic factors and survival after pulmonary resection of metastatic renal cell carcinoma. Eur Urol.2005;48(1):77-82.

16. Escudier B, Porta C, Schmidinger M, Rioux-Leclercq N, Bex A, Khoo $V$, et al. Renal cell carcinoma: ESMO Clinical Practice Guidelines for diagnosis, treatment and follow-up. Ann Oncol.2016;27(Suppl. 5):v58-v68.

17. Naito S, Kinoshita H, Kondo T, Shinohara N, Kasahara T, Saito $\mathrm{K}$, et al. Prognostic factors of patients with metastatic renal cell carcinoma with removed metastases: a multicenter study of 556 patients. Urology.2013;82(4):846-51.

18. Meacci E, Nachira D, Congedo MT, Porziella V, Chiappetta M, Ferretti $G$, et al. Lung metastasectomy following kidney tumors: outcomes and prognostic factors from a single-center experience. J Thorac Dis.2017;9(Suppl 12):S1267. doi: 10.21037/jtd.2017.05.04

19. Chen F, Fujinaga T, Shoji T, Miyahara R, Bando T, Okubo K, et al. Pulmonary resection for metastasis from renal cell carcinoma. Interactive cardiovascular and thoracic surgery. Interact Cardiovasc Thorac Surg.2008;7(5):825-8. doi: 10.1510/icvts.2008.181065.

20. Eggener SE, Yossepowitch 0, Kundu S, Motzer RJ, Russo P. Risk score and metastasectomy independently impact prognosis of patients with recurrent renal cell carcinoma. J Urol.2008;180(3):873-8. doi: 10.21037/atm.2019.11.139

21. Bianchi M, Sun M, Jeldres C, Shariat S, Trinh Q-D, Briganti A, et al. Distribution of metastatic sites in renal cell carcinoma: a population-based analysis. Ann Oncol.2012;23(4):973-80. doi: 10.1093/annonc/mdr362.

22. Hamieh L, McKay RR, Lin X, Moreira RB, Simantov R, Choueiri TK. Effect of metformin use on survival outcomes in patients with metastatic renal cell carcinoma. Clin Genitourin Cancer.2017;15(2):221-9. doi: 10.1016/j.clgc.2016.06.017.

23. Derosa L, Izzedine H, Albiges L, Escudier B. Hypertension and angiotensin system inhibitors in patients with metastatic renal cell carcinoma. Oncol Rev.2016;10(2):298. doi: 10.4081/oncol.2016.298 


\section{SUPPLEMENTARY TABLE S1.}

Patient characteristics, unmatched cohort

\begin{tabular}{|c|c|c|c|}
\hline Variable & Incomplete Metastasectomy & No Metastasectomy & $P$ Value \\
\hline No. patients & 146 & 1221 & \\
\hline Median age at diagnosis (IQR) & $63.5(56-69)$ & $64(56-72)$ & $0.002^{\mathrm{a}}$ \\
\hline Over 65 years old at diagnosis (yes vs no), \% & 42.5 & 48.7 & 0.152 \\
\hline Male, \% & 78.8 & 73.5 & \multirow{2}{*}{0.167} \\
\hline Female, \% & 21.2 & 26.5 & \\
\hline Median follow-up, months (IOR) & $26.5(16-54)$ & $19(9-36)$ & $<0.0001^{a}$ \\
\hline $\begin{array}{l}\text { Time from primary tumor to metastasis, } \\
\text { median, months (IQR) }\end{array}$ & $13.0(0-56)$ & $4.8(0-22)$ & $<0.0001^{a}$ \\
\hline Over 1 year from primary tumor to metastasis, \% & 50.7 & 33.3 & $<0.001$ \\
\hline \multicolumn{4}{|l|}{ Pathological T-stage, $\%$} \\
\hline $\mathrm{T} 1$ & 24.7 & 16.5 & \multirow{5}{*}{0.0004} \\
\hline $\mathrm{T} 2$ & 15.8 & 12.7 & \\
\hline T3 & 48.0 & 62.4 & \\
\hline $\mathrm{T} 4$ & 5.5 & 6.6 & \\
\hline $\mathrm{Tx}$ & 6.2 & 1.9 & \\
\hline Clear cell RCC (ref: yes), \% & 83.6 & 81.1 & 0.467 \\
\hline Synchronous metastasis (ref: yes), \% & 39.0 & 44.9 & \multirow{2}{*}{0.179} \\
\hline Metachronous metastasis (ref: yes), \% & 61.0 & 55.1 & \\
\hline Had a nephrectomy (ref: yes), \% & 100 & 100 & - \\
\hline \multicolumn{4}{|l|}{ Location of metastasectomy ${ }^{b}, \%$} \\
\hline Lung & 19.9 & - & - \\
\hline Bone & 29.5 & - & - \\
\hline Liver & 2.7 & - & - \\
\hline Brain & 14.4 & - & - \\
\hline Lymph node & 10.3 & - & - \\
\hline Endocrine & 13.0 & - & - \\
\hline
\end{tabular}

\section{Number of organs with metastasis, \%}

${ }^{a}$ Wilcoxon rank-sum test. ${ }^{b}$ Patients with multiple locations were counted in all specific locations; the total can exceed $100 \% .{ }^{c}$ Fisher exact text. 


\section{SUPPLEMENTARY TABLE S1.}

Patient characteristics, unmatched cohort, Cont'd

\begin{tabular}{|c|c|c|c|}
\hline Variable & Incomplete Metastasectomy & No Metastasectomy & P Value \\
\hline 1 & 72.6 & 68.4 & 0.299 \\
\hline$\geq 2$ & 27.4 & 31.6 & \\
\hline \multicolumn{4}{|l|}{ Metastasis location ${ }^{b}, \%$} \\
\hline Lung & 39.0 & 54.7 & 0.0003 \\
\hline Bones & 27.4 & 15.7 & 0.0004 \\
\hline Liver & 8.2 & 9.8 & $0.554^{\mathrm{C}}$ \\
\hline Brain & 7.5 & 1.5 & $<0.0001^{c}$ \\
\hline Lymph nodes & 16.4 & 27.9 & 0.003 \\
\hline Endocrine & 15.1 & 13.3 & $0.55^{\mathrm{C}}$ \\
\hline \multicolumn{4}{|l|}{ Systemic treatment during follow-up, $\%$} \\
\hline First-line & 71.2 & 73.5 & 0.565 \\
\hline Second-line & 33.7 & 30.1 & 0.456 \\
\hline Radiation therapy at index date and during follow-up, \% & 57.5 & 36.3 & $<0.0001$ \\
\hline \multicolumn{4}{|l|}{ Number of metastasectomy, \% } \\
\hline 1 & 82.2 & - & \multirow{2}{*}{0.587} \\
\hline$\geq 2$ & 17.8 & - & \\
\hline \multicolumn{4}{|l|}{ Comorbidities, $\%$} \\
\hline Hypertension (ref: yes) & 54.1 & 52.0 & 0.631 \\
\hline Diabetes (ref: yes) & 28.1 & 21.5 & 0.069 \\
\hline Hypercholesterolemia (ref: yes) & 24.0 & 19.7 & 0.219 \\
\hline Coronary artery disease (ref: yes) & 7.5 & 10.7 & 0.243 \\
\hline Cardiovascular disease (ref: yes) & 11.0 & 9.3 & 0.506 \\
\hline Smoker (ref: yes) & 4.8 & 4.1 & $0.689 \mathrm{c}$ \\
\hline Obesity (ref: yes) & 3.4 & 3.8 & $0.836^{c}$ \\
\hline \multicolumn{4}{|l|}{ Charlson Score Index, \% N = 1374} \\
\hline 0 or 1 & 67.7 & 50.2 & \multirow{2}{*}{0.0001} \\
\hline More than 1 & 32.3 & 49.8 & \\
\hline \multicolumn{4}{|l|}{ Propensity score quintiles } \\
\hline First & 69.2 & 87.5 & \multirow{4}{*}{$<0.0001$} \\
\hline Second & 25.3 & 11.6 & \\
\hline Third & 5.5 & 0.9 & \\
\hline Fourth & - & - & \\
\hline
\end{tabular}

${ }^{a}$ Wilcoxon rank-sum test. ${ }^{b}$ Patients with multiple locations were counted in all specific locations; the total can exceed $100 \% .{ }^{c}$ Fisher exact text. 


\section{SUPPLEMENTARY FIGURE 1 A.}

Overall survival for patients with IM by metastasectomy site

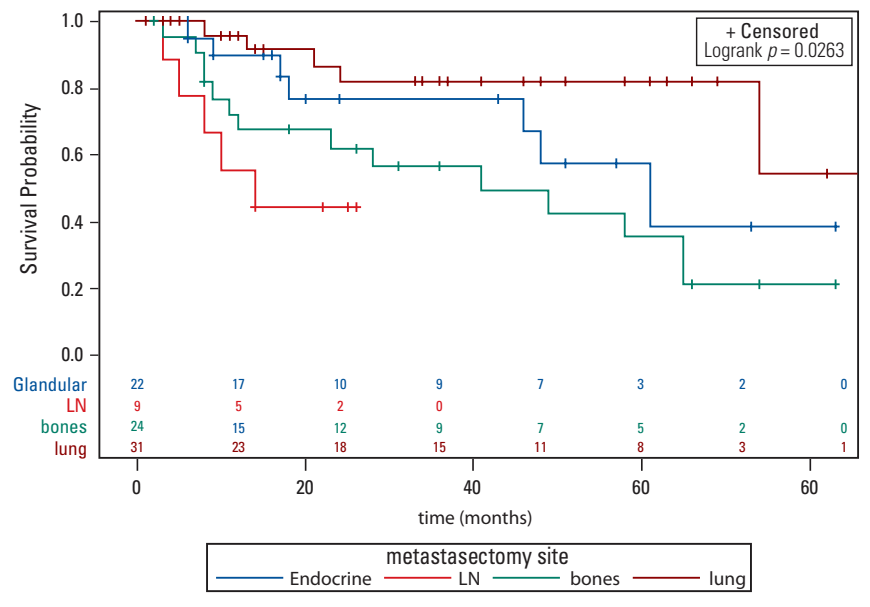

\section{SUPPLEMENTARY FIGURE 1B.}

Time to first line systemic treatment for patients with IM by metastasectomy

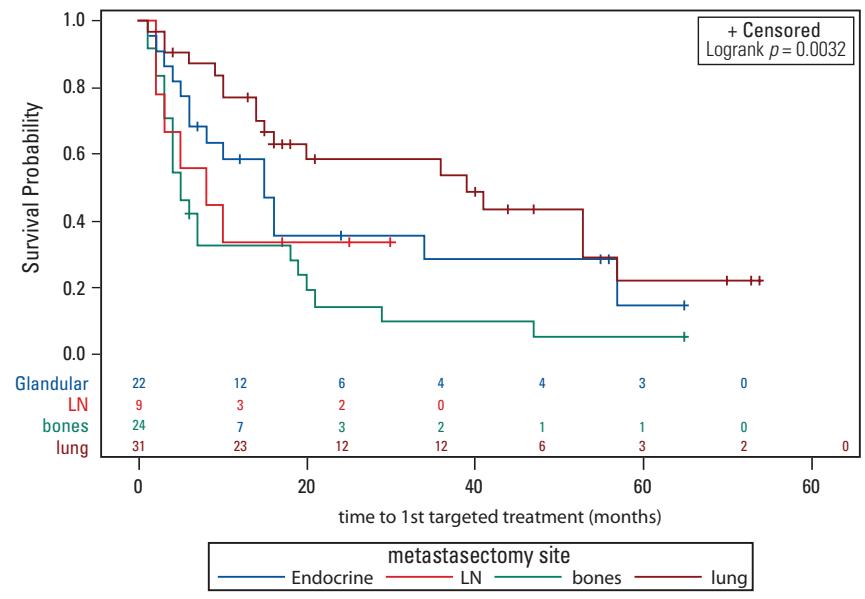

